\title{
Two-way Mirrors: Dancing in the Zoomosphere
}

Siobhan Murphy, University of Melbourne

\section{Abstract}

Dan Graham's work with mirrors and installation from the 1970s provides an unexpected launchpad for understanding the structures underlying perception in Zoom interfaces. The article explores the intricacies of Graham's two-way mirrors as a means to articulate perceptual experiences of dancing in the Zoomosphere, with examples from teaching, choreographing and performing via Zoom during Covid 19 isolation conditions.

Keywords: Zoom, dance, performance, choreography, mirror, two-way mirror, Dan Graham, perception, Covid-19, "End Meeting for All", "The World's Smallest Stage"

The phenomenon of experiencing performance online via video conferencing platforms is very much a 2020 experience, be it for teaching, learning, choreographing, performing, or as an audience member. However, some of the structural properties of experiencing performance via Zoom can be glimpsed in earlier decades. In this article, I compare Zoom experiences of performance with visual artist Dan Graham's work with mirrors from the 1970s, which he describes as situations in which audiences experience their own "perceptual processes."1 Graham's work from the 1970s is hauntingly prescient of the structures underlying perceptual and performative experience in Zoom: his use of two-way mirrors lays bare the intertwining of multiple perspectives in perception. I discuss Graham's work in the context of performance because the structures of perception that he reveals are currently being experienced in an intensified mode during the Covid-19 crisis, with performance being shifted to online conferencing platforms: we are dancing in the Zoomosphere, a word I use to designate the virtual space created by video conferencing interfaces.

I use examples from Graham's oeuvre to clarify perceptual experiences in Zoom scenarios, so as to point towards some of the ways in which performing artists have harnessed Zoom's strengths and sidestepped its shortcomings. The Zoom scenarios I explore range from my own experience of teaching dance students, student responses to choreographic tasks, choreographers' observations while transitioning to virtual rehearsals, dance works created via Zoom and bearing traces of the medium, and finally 
a theatre performance that utilised Zoom tropes as performance material and as a platform. These different scenarios have vastly different aims, but all share some perceptual similarities through their varying degrees of enmeshment in the Zoomosphere. I unpack various ways that each Zoom scenario relates to the perceptual structures laid bare in Graham's experiments with two-way mirrors.

\section{Perceptual challenges in the Zoomosphere}

As I will describe, Graham uses two-way mirrors with the overt aim of challenging the audience's perceptual habits. When dancing in the Zoomosphere, the same perceptual habits are unsettled, but this is not always the aim of the experience. The final Zoom scenario I analyze in this article, that of theatre company Forced Entertainment's Zoombased performance End Meeting for All (2020) does indeed set out to challenge viewers' perceptual and spectatorial habits. Other examples I discuss in this article have different aims, such as developing and rehearsing material together through the digital interface. Trying to focus on such tasks while one's perceptual habits are constantly unsettled by the structural properties of the medium can be baffling, frustrating, and plain exhausting. While Zoom has provided a lifeline for some semblance of continuation of embodied practices during the Covid-19 crisis, it is useful to explore the particular communicative difficulties it brings so as to elucidate ways forward.

Zoom fatigue is of course not exclusive to performance experiences conducted through the internet. Workplaces in many parts of the globe have shifted to working from home via Zoom or other platforms since the Covid-19 pandemic took hold in early 2020. The abruptness of this change in workplace habits provided a sharp perspective from which to notice the surge of fatigue experienced by workers and students alike. Andrew Hines, a computer scientist specializing in speech, audio, and video signal processing, notes that some of the fatigue comes from the crude quality of the audio signal reaching our ears in a Zoom conference. ${ }^{2}$ Rather than the dulcet tones of a radio presenter, voices in Zoom can sound scratchy, and this is often combined with unexpected, high-pitched frequencies from the squeak of a chair or sipping a cup of tea. Without the spatial context of a physical room where we can make sense of sound through its location, high-pitched sound intrusions signal our brain to be on high alert, making us stressed. ${ }^{3}$

Hines also notes we miss the meta-information supplied by body language in conversation that would help us intuitively know when it is our turn to speak, for example, so our brains work extra hard to fill in the gaps. ${ }^{4}$ Noise gates (technological devices deployed by software systems to prevent audio feedback), mean that only one voice can be heard at a time, so when multiple participants attempt to speak at once, segments of speech get cut off, leaving meaningless fragments floating in the void. Organizational behaviorist André Spicer similarly points to the information that is missing in a Zoom situation such as smell and spatialization. ${ }^{5}$ As our brains are partially 
occupied compensating for what is missing, we absorb less of what is actually being communicated.

Jeremy Bailenson, director of Stanford University's Virtual Human Interaction Lab, suggests that the excess of mis-matched communicative cues in video conferencing is overwhelming and makes it hard to achieve synchrony-and yet we are hard-wired to get 'in sync' with one another and our brains work overtime to achieve it. ${ }^{6}$ The physiological and emotional toll is greater the poorer the internet connection is. Spicer notes that if the line is glitchy, involving lags and freezes, this activates unconscious bias. For example, even a very short lag creates the perception the presenter is less friendly. If the video quality is poor, we become more cautious in our communication. ${ }^{7}$

In an interesting amplification of Graham's experimentations with two-way mirrors, seeing ourselves replicated in the Zoom 'mirror' while attempting to communicate personal content has been shown to reduce confidence and cause stress. ${ }^{8}$ Organizational psychologist Marisa Shuffler notes that it is difficult not to look at one's own image when in a Zoom conference, and seeing oneself on camera brings a requirement to perform which is stressful for many. ${ }^{9}$ Organizational behaviorist Gianpiero Petriglieri suggests that larger group conferences exacerbate performativity, commenting that a large video call "is like you're watching television and television is watching you."10

Petriglieri also draws attention to the way Zoom conferencing in isolation causes a collapse of zones that were once discrete: home, work, and social spaces. Different contexts draw out different aspects of the self, and the difference is healthy. Collapsing the different aspects of self into the one virtual zone has been shown to induce negative feelings. ${ }^{11}$ In some circumstances, the domesticity of dancing in Zoomosphere provides useful informal connection at a time of anxiety and crisis. In an article for the Washington Post, veteran choreographer Mark Morris chuffs, "It's so nice to see everybody's crap that they have," while directing a new work via Zoom, his dancers dancing in their living rooms while using bathrooms and hallways as stage wings. ${ }^{12}$

In my own Zoom teaching with dance students at the Victorian College of the Arts in Melbourne, pets on laps became a motif that provided humorous continuity from one session to another. Concurrently, amid the general overwhelm of trying to scan across 27 faces, I would also notice with a pang the vastly different socio-economic circumstances in which students were attempting to learn. Some would receive a cup of tea mid-way through a lecture, lovingly delivered by a parent. Others were perched on the end of their bed, the only private space in a busy shared house. Others were alone in blank rooms in tiny apartments, international students who had not had a chance to furnish their dwelling before isolation restrictions began. The Zoom grid both brings people together and underlines their separateness. In a later livestream of his company's work created via Zoom, Morris commented that during rehearsals, seeing all the dancers in their own distinct rectangular squares on the Zoom grid accentuated his 
sense of them as individuals - unlike his prior perception of them as a group in the physical studio. $^{13}$

The potential complications studied by behavioral psychologists and communications experts are real for those dancing in the Zoomosphere. Video conferencing is nevertheless currently a vital platform for financial survival, group experience and artistic development for performing artists, much as we may long for the resumption of 'live' or 'real' kinesthetic interaction. I will now outline Dan Graham's experimental perceptual processes as a way to explore the structure of the two-way mirror, but also to move towards the idea that artists of various kinds are uniquely placed to experiment with the new medium of Zoom. Artists are experts, in their given modality, in experimenting with human perception.

\section{The perceptual structures of Dan Graham's two-way mirrors}

In her volume Mirror Affect: Seeing Self, Observing Others in Contemporary Art, art historian Cristina Albu analyzes visual art works that contain mirrors, live video feedback, and sensors from the 1960s onwards, as situations that provoke consideration of the interpersonal nature of perception. Albu argues that in the 1960s, mirrored surfaces, live video feedback, and sensors were used to challenge the autonomous materiality of the art object, an aim shared by other art movements at the time such as Fluxus and happenings. For Albu, in the 1970s the use of mirrors shifted, to instead critique the supposed privacy and neutrality of aesthetic experience, literally showing viewers that they were in fact part of a collectivity: "An increasing number of artists, including Vito Acconci, William Anastasi, Judith Baca, Daniel Buren, Peter Campus, Dan Graham, Lynn Hershman, Bruce Nauman, and Peter Weibel, designed visual systems that incorporated competing reflective surfaces that would vie for participants' attention and enable critical distance from the all-engulfing conditions of the society of the spectacle."14 Albu provides a Foucauldian critique of the operation of power in mirrored environments, detailing the duality of surveillance and agency. However, this aspect of her analysis is beyond the scope of this article, in which I largely focus, in a more basic way, on the processes of perception at play in Graham's work.

Dan Graham's 1975 performance work Performance/Audience/Mirror illustrates the impossibility of maintaining a neutral standpoint during perception. ${ }^{15}$ Instead, his performative actions cumulatively show a constant intertwining of multiple viewpoints, brought about by the operations of social dynamics made manifest through the formal device of the mirror. The work comprises a mirror on one wall, with viewers facing it, and Graham performing between the mirror and the viewers. The work proceeds through four stages, each containing unrehearsed observations of small physical actions. First, Graham describes his physical stance and minute actions while directly facing the audience. Next, he shifts to describing the audience while facing them. Third, he turns to face the mirror and describes himself while observing himself in the mirror. 
Finally, while still facing the mirror, he describes the audience as he observes them in the mirror. Proceeding from the proximal observation of his own movement to the more distanced observation of others' reflections, Graham appears as a vector through whom observations pass, rather than as a performer who originates the event. The vocal cataloguing of movements has a quasi-scientific quality to it, inviting impressions of neutrality.

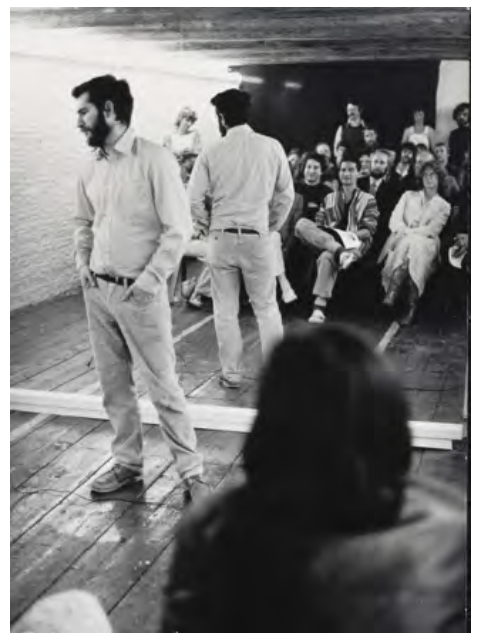

Dan Graham, Performer/Audience/Mirror, 1977. De Appel Arts Center, Amsterdam. Courtesy of Dan Graham and Marian Goodman Gallery, Paris.

However, on closer examination the work discloses the impossibility of neutral observation of others: what Graham notices and voices about himself and others' movements is necessarily laced with the particularities of his own subject position. Moreover, by the fourth stage of the work, it becomes increasingly difficult to identify who is the performer and who is the audience. It may be Graham who is noticing and naming movements, postures, and attitudes of others as he perceives them in the mirrored reflection, but by this point, the audience is accustomed to the performative set-up and can choose to be still or to contribute movement to be noticed and named. Graham's voiced observations are necessarily temporally offset from the visual experience: a lag emerges between vision and description. Audience members might observe themselves instantaneously through the visual medium of the mirror, then hear themselves described moments later through Graham's observation of them. This dynamic may start to sound familiar to those who have spent immense amounts of hours in Zoom conferencing in recent months. ${ }^{16}$ The correlations deepen in a subsequent work by Graham.

In his first architectural installation, Public Space, Two Audiences (1976), Graham used a two-way mirror, acoustically insulated and fitted snugly to the walls of the gallery space, dividing it into two rooms entered through each end. One of the rooms contains an additional mirror on the back wall. The audience sees a reflection of themselves, as well as seeing through the glass to the other side where other audience members are doing the same dual looking. Effectively the viewers on either side become performers for 
each other, at the same time as they watch themselves watching. Graham describes it thus: "they are in a showcase situation, and what's on display is peoples' perceptual processes [...] people see each other in a group seeing themselves and seeing each other."17 Importantly, there is no vantage point on the work that sits outside this structure: one has to be in either room in order to experience it. This suggests the impossibility of escaping surveillance: in order to view, one has to be on view for others.

\section{PUBLIC SPACE / TWO AUDIENCES}
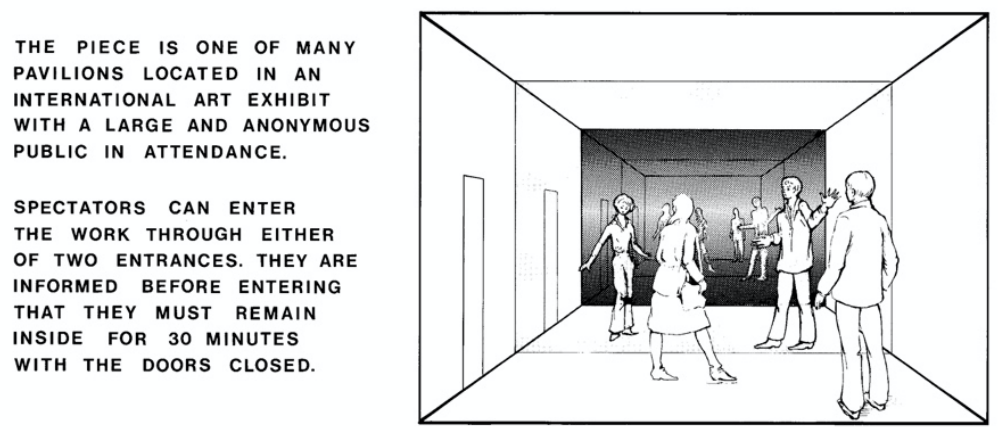

EACH AUDIENCE SEES

THE OTHER AUDIENCE'S

VISUAL BEHAVIOR, BUT

IS ISOLATED FROM THEIR

AURAL BEHAVIOR. EACH

AUDIENCE IS MADE MORE

AWARE OF ITS OWN

VERBAL COMMUNICATIONS

IT IS ASSUMED THAT

AFTER A TIME, EACH

AUDIENCE WILL DEVELOP

A SOCIAL COHESION AND

GROUP IDENTITY.



Dan Graham, diagram of Public Space/Two Audiences, 1976. Courtesy of Dan Graham and Marian Goodman Gallery, Paris.

Albu notes that far from being enamored by mirrors, Graham was suspicious of them, considering them to be "symbols of stagnation and control."18 Rather than the straightforward narcissism implied by simple mirrors, Graham's two-way mirrors plunge the viewer into intense visual relationships with others, enmeshing self and other in a complex web of imagery. Albu argues that Public Space, Two Audiences promoted a sense of belonging to a "diffuse collectivity" generating "affective alliances" that served to reach through the mirror interface. ${ }^{19}$ Although there was no outside vantage point, the ambiguity of the two-way mirror allowed curious viewers to modify their images relative to others through proximity and distance, and to gain agency through instigating gestural threads of non-verbal communication. Viewers initially looked at 
each other through the mirror and then started to creatively interact with each other's gestures. In the context of Graham's installation, Albu calls this movement impulse "affective attunement." ${ }^{20}$ Precise accounts of the movement interaction are not available, but it can be surmised that an element of mimesis came into play. That is, the basic impulse to achieve synchrony with another person gives rise to imitation of gesture, posture, and facial expression. As discussed, in the Zoom situation the medium's imperfections frustrate our attempts at synchrony, resulting in fatigue. In the context of Public Space, Two Audiences the attempt at synchrony is reliant on a viewer on the other side choosing to play along, emphasizing the contingency of communication. In describing the quality of affective attunement, Albu cites Graham's own formulation of the experience: "While the glass-partition on one hand places a distance between opposing spectators, on the other hand, the co-presence on the mirror of the two groups' bodies and the visual image of their process of looking make for an extreme visual inter-subjective intimacy." ${ }^{\prime 21}$

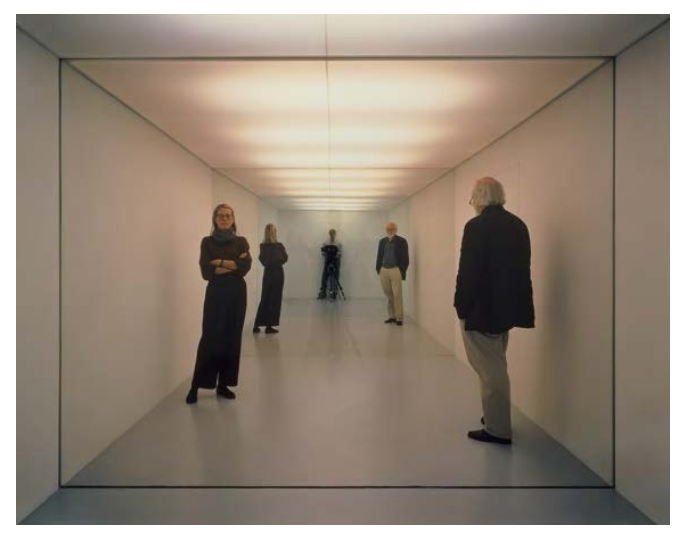

Dan Graham, Public Space/Two Audiences, 1976. Courtesy of Dan Graham and Marian Goodman Gallery, Paris.

In Public Space, Two Audiences, the two-way mirror created two white zones that were identical except that one housed an additional mirror. Dancing in the Zoomosphere, Graham's two rooms become the living rooms or even bedrooms of performers, directors, choreographers, teachers, and audience members. Gone is the supposed neutrality of the white-walled gallery space: instead, the Zoom interface opens directly onto intimate private spheres. As I will explore below, this casts Graham's "extreme visual inter-subjective intimacy" in a new light.

\section{Performative interactions in the Zoomosphere}

When using Zoom for dance experiences, the video interface has some structural properties similar to the two performative installation works of Graham's I have described thus far. I suggest that the Zoom interface becomes something like Graham's two-way mirror: we see the other performer(s) at the same time that we see ourselves seeing them. Take for example, the situation of a choreographer working one-on-one with a dancer via Zoom. If the dancer wants to remain in the camera's frame so that the choreographer can see her, she needs to dance in relation to the image of herself dancing, in a sense dancing to her own image at the same time that she sees the image 
of the choreographer observing her. Of course, trained dancers are accustomed to moving with equal attention to sensation and form, but the crude mirror mechanism of Zoom heightens the dual operation.

In a conversation on 11 September 2020 about the communication of choreographic ideas via Zoom during lockdown, Melbourne choreographer Sandra Parker noted that the experience prompted her to ask: "What do you need to know? Not everything." She was referring to developing a new piece with dancers she has worked with extensively over many years. If she gave a task, and the dancers slipped out of the camera's frame in the act of responding to the task, she filled in the information that was missing:

I am so practiced at looking at the moving body that I can tell, from the angle of a shoulder, for example, where the arm might be emanating into the offscreen space. This practiced way of predicting bodily movement driven by rhythm, space and the body's weight was coupled with my memory of the movement already set. I can just keep going because I don't have to worry that I need to see everything. [...] how much of the material that we're working with is actually visual anyway?

I wondered if Zoom perhaps forces this re-alignment of perceptual priorities, if, in the absence of visual information, other perceptual modes come to the fore to allow the choreography to continue. Parker agreed: "yes, otherwise you'd be constantly asking the dancer to re-orientate, to put themselves in the center of the frame." Perhaps Zoom here functions as a two-way mirror as in Graham's work, but also as a container for holding an embodied situation in place, in the way one might speak of 'holding the room' in a group improvisational context.

The situation Parker describes is very much predicated on knowing the dancers well, on a shared prior history that informs the Zoom encounter. The situation of being plunged into teaching via Zoom with students one does not know well, if at all, provokes a different set of priorities to emerge. In teaching choreography to first year students at the Victorian College of the Arts in Melbourne, who had only had two weeks of face-toface learning prior to going into isolation and remote learning, Parker focused on problematizing the idea of the visual when copying movement. Given that students were spending so much of their time looking at dance on screens, Parker asked them to interrogate what they were looking at and how it could be put to work choreographically, opening up expectations of what could be derived from the flat visual plane.

Working in pairs via breakout rooms in Zoom, some students chose to incorporate the lags and glitches brought about by their variable internet speeds and insert these artifacts into their choreographies. This is a potent example of Susan Broadhurst's claim that mediated contexts spawn alternative ways of constructing meaning. ${ }^{22}$ Students Aimee Raitman and Samakshi Sidhu described their approach thus: 
Our short video piece reflects our surrender to the volatile and unpredictable cyberspace we have inhabited over the past 6 months. When we first began working together on this we found that many of our choreographic and movement choices unintentionally mimicked the frustrating glitches we often face on zoom during our daily practices in uni. Rather than trying to fight these impulses, and imagine ourselves back in a studio setting, we wanted to embody our new reality, and find comfort in the perfect, yet sometimes frustratingly imperfect, pixels on our screen. Often we see a glitch as being a difficulty that needs to be overcome during zoom rehearsals, so, through filming our piece [...] we transformed these chance occurrences into deliberate and considered events, reimagining them as vital to the choreography. ${ }^{23}$

The students' experience resonates with Kerry Francksen's descriptions of mediated environments from the perspective of the dancer. She discusses a situation in which the dancer improvises live in relation to a projected image of that same improvisation, digitally manipulated or treated. It is clearly a different set-up to working choreographically via Zoom, but the insights are strikingly resonant:

the dancers were not watching their screened presence, they were moving with the sense that their fleshy bodies were enveloped in a strange duet, which happened to cross between the live and the digital. In this way, the digital was not only informative of how and why the dancers made certain movement choices, but it also became entangled into her experience of moving. ${ }^{24}$

Francksen notes that over time, the continued experience of performing in this intertwined way - this 'strange duet'-developed in the dancers a sense of subjectivity that inhabits the virtual and the material simultaneously. ${ }^{25}$ Francksen is referring here to Matthew Causey's concept of the biovirtual. ${ }^{26}$ Biovirtual kinesthesia is an apt description of the skill developed by dancers like the first year students Parker worked with, who spent large parts of every day learning dance technique and composition via Zoom from March to November 2020, constantly in a 'strange duet' with their virtual selves imaged via the Zoom camera.

Working with the same group of first year students some months later under renewed conditions of lockdown and curfew, Parker began their choreographic process by asking them to look anew at the world they were in. They cataloged the spaces in which they were choreographing, their bedrooms and living rooms, in the style of Georges Perec's auditing of the contents of his desk, to derive material that could be sampled, re-contextualized, and repeated in duet format. Similar to the students' approach of incorporating video artifacts into their choreography, this task recasts or subverts the difficulties of the medium. The confined physical spaces of lockdown, and the Zoomosphere's collapsing of the discrete spaces for learning, sleeping, eating, and 
dancing, are countered by the expansive, generative task of rigorously auditing one's surroundings, mining it for content. This information is then offered to a partner as material in the creation of a duet to be performed together via Zoom.

Thinking back to Graham's "extreme visual inter-subjective intimacy" brought about through the two-way mirror, Parker's deliberate use of the intimate rooms of the Zoomosphere resonates with Robert Enright and Meeka Walsh's description of Graham's pavilions of glass and mirror. They describe the "double hook" operating in his perceptual structures, noting that the viewer is simultaneously "accessing information and being information." ${ }^{27}$

In conditions of lockdown, dancers in the Australian Dance Theatre (ADT) were paired up with independent choreographers and musicians to create new short works via video conferencing. ${ }^{28}$ The World's Smallest Stage matched 10 choreographers with 12 dancers and 10 composers for the new works, made in living room sized areas of $2 \mathrm{~m} \mathrm{x}$ $2 \mathrm{~m}$. The works were later converted into live performances at the Odeon Theatre in Adelaide in October 2020, but their initial public outing was as pre-recorded single shot videos made available through the company's Facebook page several months prior, and it is this earlier public sharing that is of interest here.

The videos display a range of traces of the video conferencing platform that was their mode of production: to varying degrees, these traces have been incorporated into the aesthetic of the works. ADT is renowned for the athleticism of its dancers, so one of the primary traces of the Zoom mechanism was the tight and often tense physical confinement of the dancers. Additionally, these are not dances shot from multiple angles and edited into screendance works. The single point of view for most of these videos echoes the choreographer's perspective of viewing each dancer from the mutual position of social isolation via Zoom or other video conferencing software. The point of view is that of a computer's camera propped on a chair, angled to capture all the action. The use of available light, or choice to restrict natural light by closing blinds or curtains, is similarly an aesthetic familiar to those who have spent many hours on Zoom during the health crisis.

Theatre scholar William Peterson interviewed some of the dancers and choreographers involved in the project to understand how choreographic communication shifted in the transition to the remote situation. ${ }^{29}$ From the dancers' perspective, a common theme was that working remotely via video conferencing honed a sharper experience of what was happening 'on the inside,' meaning the feeling of the movement rather than the look of it. Ultimately what stood out to Peterson was the impact of the reduced scale of movement. Peterson recounts dancer Rowan Rossi's comment that when he moves, he usually tries to take up as much space as possible. Working in a confined space meant he had to occupy space differently, shifting the scale of communication by "embellishing smaller gestures." 
Rossi, in "Shapeless/Formless" by Felicity Boyd, dances with rosy velvet cushions in the shape of spheres and a pyramid. ${ }^{31}$ These enigmatic, domestic objects become unlikely dance partners and a means of extending the reach of Rossi's gestures, in the absence of large-scale movement. As with many of the pieces in The World's Smallest Stage, the camera's point of view is that of the absent choreographer: we see the dancer's interior domestic space as though in rehearsal, and with it comes a hint of voyeurism, recalling Graham's claim that two-way mirrors engender an extreme visual inter-subjective intimacy. As if to counter this effect, Boyd and Rossi have attempted to neutralize or clean up the domestic a little, hanging a white sheet like a theatre scrim across a wall that may have been too domestic, distracting, or simply private.

By contrast, Matt Shilcock's choreography "Preliminary" with Kimball Wong utilises the ready-made aesthetic of an emptied-out garage, a space used by others across the globe as a makeshift studio during the health crisis. Wong unpacks worn, tessellated foam tiles to create the requisite $2 \mathrm{~m} \times 2 \mathrm{~m}$ space, and to allow movement that a concrete surface would inhibit. Accompanied by electric guitar, his movements are reminiscent of a caged animal pacing and raging against its restriction. Yet he is complicit in his restriction, shown in the compliance with which he folds up his tessellated squares at the end of the piece, reducing his available space to a messy pile of foam tiles on which he squats, energy spent for now. As Peterson noted in Zoom conversation with me on 9 December 2020, the muscular tension within some of these performances of restriction "speaks to our own experiences of containment" during recent periods of lockdown.

End Meeting for All, by UK theatre company Forced Entertainment, similarly speaks to the viewer's own experiences of containment, but in an entirely different way. The project took shape in the early days of lockdown in the UK in the form of three short, improvised Zoom meetings, streamed by PACT Zollverein from 28 April until 30 June $2020 .^{32}$ With the company's planned rehearsals and performances put on indefinite hold, the company members dialled into Zoom meetings from London, Sheffield and Berlin. Although the meetings were not initially intended as creative developments, director Tim Etchells soon realised that company members were "slowly starting to understand the Zoom grid as a kind of stage." ${ }^{33}$ The work trades on viewers' familiarity with the vagaries of the Zoomosphere, using its perceptual structures as primary content. The three episodes are Zoom meetings recorded in one take in gallery view, each performer occupying a rectangle in the grid.

The episodes appear as rehearsals for a work that never quite arrives. Some performers spend entire episodes on the periphery of the action, waiting for a signal from the others that they can be seen and heard. Claire Marshall, on the other hand, has worked up some melodramatic content to try out, donning a messy grey wig and telling the others she is using it to indicate that she has been in quarantine a very, very long time. Claire's desire to get some dramatic content up and running and her frustrated attempts to find an audience for it is the through line of the fragmentary episodes. However hard and by 
whatever means Claire tries to reach through the Zoom interface towards her coperformers, Cathy Naden thwarts her, declaring that her screen is frozen or that she (Claire) cannot be heard at all. Regarding Graham's Performance/Audience/Mirror, I described how by the fourth stage of the work, the roles of performer and audience became productively ambiguous and intertwined. In End Meeting for All, Cathy's usurping of Zoom's communicative failures to thwart Claire's melodrama begs the question of whether a performance exists at all if the attendees of one's Zoom meeting cannot or will not bear witness to the event.

Theatre scholar Barbara Fuchs comments on the work's use of Zoom for metatheatrical purposes: the various narratives carried forward by the performers provide the opportunity to comment on how performance communicates and miscommunicates, and the Zoom medium's glitches, lags and tedium are used to explore this. Further, Fuchs notes:

The metatheatricality is in fact enhanced by Zoom, as the fourth wall is multiplied several times over. In their windows, each performer becomes a separate audience for the others, with varying degrees of engagement. In addition to thematizing the technological failures of communication, the grid reminds us that some participants are just not interested or simply unable to engage with the shared project. ${ }^{34}$

A recurrent theme of the work is sadness, with Terry O'Connor drinking gin for the duration and commenting that it is good to let the sadness out. Cathy says she is feeling sad and splashes water under her eyes to let the other performers know just how sad she is. While the work's claustrophobia and pathos undoubtedly speak to others' experiences of lockdown, the strong emphasis on these emotions paradoxically highlights the privilege involved in being able to socially isolate.

Etchells commented that "the grid of screens brought together different partially connected realities in different cities, the screen a kind of membrane or imperfect portal between worlds" (2020). Regarding Graham's Public Space: Two Audiences, I discussed Albu's concept of affective attunement as a descriptor of the relationships between viewers provoked by Graham's two-way mirror. Albu suggests that affective attunement with others across the mirrored interface is the means by which the work gives rise to a diffuse collectivity. In End Meeting for All, the collectivity enacted by the performers in the Zoom situation is particularly diffuse. At times, performers attempt to tune in to another's state of mind by reading each other's facial cues and posture, noticing when someone seems distressed, for example. At other times, communicative cues are deliberately ignored, calling to mind the fact that in Graham's installation, affective attunement relied upon another viewer choosing to join the kinesthetic conversation from the other side of the mirror. 


\section{Conclusion}

In this article, I have given some examples of ways that artists have experimented, in their given modalities, with structures of perception. Graham's oeuvre seeks to expand perceptual awareness beyond passive, introspective contemplation of the art object. Instead, his installations oscillate between multiple perspectives and only come fully into being through the viewer's participation. As Albu suggests, such work frames "contexts for seeing ourselves seeing and acting as part of precarious collectivities." The kinesthetic collectivities of the Zoomosphere are both urgent and precarious: we are driven to join Zoom meetings at the same time that our perceptual systems struggle with them. The two-way mirrors of both Zoom interactions and Graham's installations invite reflection on the interpersonal nature of perception: ultimately, they also invite reflection on the ways subjectivity itself is sourced from and is responsive to a plethora of shifting cues and interactions. At times, they do so through failure, such as when no one responds to one's gesture in Public Space, Two Audiences, or the Zoom interface fails, leaving one's utterances stranded in the void. As Enright and Walsh remind us, in Graham's stripped back perceptual scenarios, the "process of shifting apprehension is one way of measuring the loss and rediscovery of self and other that is central to the experiential impact of Graham's pavilions, whether they are inside a gallery or outside in a garden or a sculpture park. You are involved, willy-nilly, in an architecture of selfconsciousness." ${ }^{\prime 36}$

Graham's two-way mirrors and Etchell's characterisation of Zoom as an imperfect portal between two worlds recall developmental stages long since incorporated into tacit understandings of being in a world with others. Lacan's theorisation of the mirror phase as constitutive of the infant's understanding of their relatedness to others is of course a developmental structure vastly prior to any engagement with the perceptual structure of two-way mirrors via visual art or Zoom interfaces. Two-way mirrors and Zoom are thus not only experiments with perceptual structures: they are also an invitation to remember our constitutive enmeshment with others. In the deep midwinter of lockdown, a friend messaged me a short video of her three-year-old son who was squatting in the grass looking at a chicken. Boy and chicken were both stock still, locked in each other's gaze. She asked him, "What are you doing, Ned?" to which he replied, "I'm looking at a chicken looking at me."

\section{Biography}

Siobhan Murphy is a dance artist and academic based in Melbourne. Her practice focuses on screendance works for single channel and gallery installation outcomes, with works screened in festivals and museums in cities throughout Australia and New 
Zealand, as well as London, Tokyo, Buenos Aires, Lisbon and Edinburgh. Her recent scholarly and artistic research centers on dance portraiture, with her portrait of Joanne White screened at the London International Screendance Festival in 2019, and her portrait of Alice Cummins at Melbourne's DanceLens in 2021. Her article on screendance portraiture examines the productive counterpoints of the portraiture tradition and builds toward an emergent framework for understanding how screendance portraits function. Siobhan is a Lecturer in Dance at the Victorian College of the Arts, University of Melbourne, where she teaches and supervises dance theory and practiceled research.

Email: mus@unimelb.edu.au

\section{Notes}

1 "Dan Graham: Beyond."

${ }^{2}$ Andrew Hines and Phoebe Sun, "Zoom fatigue."

${ }^{3}$ lbid.

${ }^{4}$ Ibid.

${ }^{5}$ André Spicer, "Finding endless video calls exhausting?"

${ }^{6}$ Bailenson in Betsy Morris, "Why Does Zoom Exhaust You?"

${ }^{7}$ Spicer.

${ }^{8}$ Matthew K. Miller, et al, "Through the Looking Glass."

9 Shuffler in Manyu Jiang, "The reason Zoom calls drain your energy."

${ }^{10}$ Petriglieri in Jiang.

${ }^{11}$ Ibid.

${ }^{12}$ Morris in Sarah Kaufman, "Morris, one of the world's leading choreographers"

${ }^{13}$ Morris in Mark Morris Dance Group, "Dance On!"

${ }^{14}$ Cristina Albu, Mirror Affect, 110.

${ }^{15}$ See https://youtu.be/RjiLZ_AOtOA for footage of Graham's 1975 performance and https://youtu.be/Gi7y5vDErZl for footage of the Body Cartography Project's interpretation of the piece at the Walker Center in 2009. 
${ }^{16}$ Temporally offsetting experience and perception was minutely explored by Graham in a 1974 work, Opposing Mirror and Video Monitors, though his work with video feedback is beyond the scope of this article.

17 "Dan Graham: Beyond."

${ }^{18}$ Albu, 111.

19 lbid. 138.

${ }^{20}$ Ibid. 149.

${ }^{21}$ Graham in Ibid. 149.

${ }^{22}$ Susan Broadhurst, Digital Practices, 15.

${ }^{23}$ Aimee Raitman and Samakshi Sidhu, "Presentation of student work."

${ }^{24}$ Kerry Francksen, "The Implications of Technology in Dance," 74.

${ }^{25}$ Ibid. 60.

${ }^{26}$ Matthew Causey, "General Introduction."

${ }^{27}$ Robert Enright and Meeka Walsh, "Dan Graham: Mirror Complexities."

${ }^{28}$ Australian Dance Theatre, The World's Smallest Stage.

${ }^{29}$ William Peterson, "The World's Smallest Stage."

${ }^{30} \mathrm{Ibid}$.

${ }^{31}$ ADT kindly shared the videos with me that were made publicly available on the company's Facebook page. They are no longer available for public viewing.

${ }^{32}$ Forced Entertainment, "End Meeting for All."

${ }^{33}$ Tim Etchells, "Falling into Place."

${ }^{34}$ Barbara Fuchs, "End Meeting for All."

${ }^{35}$ Albu, 1.

${ }^{36}$ Enright and Walsh. 


\section{References}

Albu, Cristina. Mirror Affect: Seeing Self, Observing Others in Contemporary Art. Minneapolis; London: University of Minnesota Press, 2016. Accessed 15 September 2020. http://www.jstor.org/stable/10.5749/j.ctt1hch81m

Australian Dance Theatre. The World's Smallest Stage. 2020. Accessed 20 December 2020. https://adt.org.au/international-choreography-centre/the-worlds-smalleststage/

Broadhurst, Susan. Digital Practices: Aesthetic and Neuroesthetic Approaches to Performance and Technology. Basingstoke: Palgrave Macmillan, 2007. https://doi.org/10.1057/9780230589841

Causey, Matthew. "General Introduction. In the After-event of the Virtual." The Performing Subject in the Space of Technology: Through the Virtual, Towards the Real. Ed. Matthew Causey, Emma Meehan, and Neill O'Dwyer. New York: Palgrave Macmillan, 2015. 1-8.

"Dan Graham: Beyond." Whitney Museum of American Art. With Commentary by Dan Graham and Chrissie lles. 2009. Video, 3:40. https://whitney.org/media/371

Enright, Robert and Meeka Walsh. "Dan Graham: Mirror Complexities." Bordercrossings 112 (December 2009). https://bordercrossingsmag.com/article/dan-graham-mirrorcomplexities

Etchells, Tim. "Falling into Place: A Note on End Meeting for All." April 2020. https://www.forcedentertainment.com/falling-into-place-a-note-on-end-meeting-forall/\#

Forced Entertainment. "End Meeting for All." 2020. Accessed December 20, 2020. https://www.forcedentertainment.com/projects/end-meeting-for-all/\#

Francksen, Kerry. "The Implications of Technology in Dance: A Dancer's Perspective of Moving in Media-Rich Environments." Digital Echoes: Spaces for Intangible and Performance-based Cultural Heritage. Eds. Sarah Whatley, Rosemary Cisneros, and Amalia Sabiescu. Cham: Palgrave Macmillan, 2018. 57-80. https://doi.org/10.1007/9783-319-73817-8_4

Fuchs, Barbara. "End Meeting for All: Zoom Theatre, Launched." Howlround Theatre Commons. 25 June 2020. https://howlround.com/end-meeting-all

Hines, Andrew and Phoebe Sun. "Zoom fatigue: how to make video calls less tiring." The Conversation. 11 May 2020. https://theconversation.com/zoom-fatigue-how-to-makevideo-calls-less-tiring-137861 
Jiang, Manyu. "The reason Zoom calls drain your energy." BBC Worklife. 23 April 2020. https://www.bbc.com/worklife/article/20200421-why-zoom-video-chats-are-soexhausting

Kaufman, Sarah. "Morris, one of the world's leading choreographers, takes dance to Zoom." Washington Post. $14 \quad$ April 2020. https://www.washingtonpost.com/entertainment/theater_dance/mark-morris-one-ofthe-worlds-leading-choreographers-takes-dance-to-zoom/2020/04/13/cc8097c47a86-11ea-b6ff-597f170df8f8_story.html

Miller, Matthew K., Regan L. Mandryk, Max V. Birk, Ansgar E. Depping, and Tushita Patel. "Through the Looking Glass: The Effects of Feedback on Self-Awareness and Conversational Behaviour during Video Chat." Proceedings of the $2017 \mathrm{CHI}$ Conference on Human Factors in Computing Systems. Association for Computing Machinery (2017): 5271-5283. https://doi.org/10.1145/3025453.3025548

Morris, Betsy. "Why Does Zoom Exhaust You? Science Has an Answer." Wall Street Journal. 27 May 2020. https://www.wsj.com/articles/why-does-zoom-exhaust-youscience-has-an-answer-11590600269

Mark Morris Dance Group. "Dance On! An Evening with the Mark Morris Dance Group." YouTube Video. 58:43 streamed live on 29 May 2020. https://www.youtube.com/watch?v=TLHnwcllAzc\&list=PLffaix81vhuPXEAC95ItiON1Kr kgPs1Qp

Peterson, William. "The World's Smallest Stage: Making Choreography Differently, Seeing Differently." Paper presented at The Australasian Association for Theatre, Drama and Performance Studies Conference. The University of New South Wales via Zoom. 3 December 2020.

Raitman, Aimee and Samakshi Sidhu. "Presentation of student work." 24 August 2020. Zoom meeting. University of Melbourne.

Spicer, André. "Finding endless video calls exhausting? You're not alone." The Conversation. 6 May 2020. https://theconversation.com/finding-endless-video-callsexhausting-youre-not-alone-137936 